\title{
Thyroid Imaging Reporting and Data System (TI-RADS): the impact of Quantitative Strain Elastography for better stratification of cancer risks.
}

\author{
Dana Stoian', Bogdan Timar², Mihnea Derban³, Stelian Pantea ${ }^{4}$, Florian Varcus ${ }^{4}$, \\ Marius Craina $^{1}$, Mihaela Craciunescu ${ }^{5}$
}

${ }^{1}$ Endocrine Unit, Department of Obstetrics Gynecology, ${ }^{2}$ Department of Medical Informatics, ${ }^{3}$ Department of Pathology, ${ }^{4}$ Department of Surgery ${ }^{5}$ Department of Microbiology, University of Medicine and Pharmacy " Victor Babes"

Timisoara, Romania

\begin{abstract}
Aims: Due to the elevated prevalence of the solid thyroid nodules in a general population, an appropriate selection of cases referred to surgery is of paramount importance. The main aim of our study was to evaluate the performance of the Thyroid Imaging Reporting and Data System (TI-RADS) as a differential diagnosis tool for thyroid nodules. Material and methods: We evaluated 174 nodules using TI-RADS risk stratification model, using conventional ultrasound and real time elastography parameters and linear multifrequency probe (Hitachi Preirus Machine, Hitachi Inc., Japan). All the nodules were classified using the TI-RADS system according to echogenicity, margins, shapes, calcification, lymph nodes, and increased strain ration. The results were compared with the pathology exam, which was considered the golden standard diagnosis. Results: The prevalence of malignant nodules was $16.7 \%$ (29 cases). The differential diagnosis performance regarding the malignant tumor for TI-RADS is appropriate for clinical use, obtaining an area under ROC curve of 0.95761 [0.8424-0.989] 95\% confidence interval. Combining TI-RADS 2, 3 and 4A as probably benign and TI-RADS 4B and 5 as probably malignant, the sensitivity, specificity, positive predictive value, and negative predictive value were $97.93 \%, 86.20 \%, 97.26 \%$ and $89.28 \%$ respectively. The overall accuracy of the method was 95.97\%.According to the American Association of Clinical Endocrinologists Guidelines, 169 cases required FNAB examination; by applying the TI-RADS scoring system, the necessity for FNAB would decrease to 74 cases. Conclusion: Quantitative strain elastography, as the $6^{\text {th }}$ parameter of TI-RADS system, adds diagnostic power to the risk stratification model.
\end{abstract}

Keywords: elastography, TI-RADS, thyroid cancer

\section{Introduction}

There is a high prevalence of thyroid nodules in the general population [1]. This prevalence increases with age [2]. The female population is more affected [3], 20 up to $70 \%$ of the female population having at least one ultrasound (US) identified thyroid nodule. Thyroid cancer has an increasing incidence $[4,5]$ due to exposure to

Received 21.02.2015 Accepted 5.05.2015

Med Ultrason

2015, Vol. 17, No 3, 327-332

Corresponding author: Mihnea Derban

Department of Pathology

9, P.M.Cristea street

300029 Timisoara, Romania

Phone: +0727804302

E-mail: mihneaderban@gmail.com medical radiation, iodine intake, obesity and insulin resistance, genetics, and inorganic phosphates [6,7], up to $15 \%$ of the total identified thyroid nodules being malignant $[5,8]$. In the presence of solid thyroid nodules the Endocrine Societies Guidelines recommend the use of ultrasound-guided Fine Needle Aspiration Biopsy (FNAB) $[1,9]$ in order to establish a proper therapeutic attitude. The American Thyroid Association (ATA) [9] stated that FNAB is the procedure of choice for the evaluation of thyroid nodules. FNAB should be performed in the presence of thyroid nodules $>5 \mathrm{~mm}$ with suspicious US findings, hypoechoic solid nodules $>1 \mathrm{~cm}$, mixed cysticsolid nodules $>1.5-2 \mathrm{~cm}$ with suspicious US findings, nodules with micro calcifications, or abnormal cervical lymph nodes. American Association Clinical Endocrinology (AACE) [10] recommend FNAB of the hypoechoic 
solid nodules $>1 \mathrm{~cm}$, nodules with malignant US findings, regardless of the size, and in patients with special malignancy history. The Korean Guideline recommends FNAB inall suspicious nodules (taller than wide shape, irregular margins, marked hypoechoic texture, micro and macro-calcifications, speculated margins, extra capsular invasion) regardless of the size and selective biopsy in probably benign nodules larger than $2 \mathrm{~cm}$ [11]. By analyzing these indications it can be observed that there are some differences for FNAB indications, especially concerning the size and the category of nodules that should be referred to FNAB [12].

Despite the significant evidence, as summarized recently [13-15], regarding the excellent diagnostic quality of real time elastography for the differential diagnosis of thyroid nodules, this method is still not included in those mentioned guidelines. Recently, some reports used with good results, a reporting data system for thyroid lesions-the Thyroid Imaging Reporting and Data System (TIRADS)- for stratifying the risk for malignancy $[16,17]$, using 12 , respectively 6 different patterns of thyroid nodules described in conventional ultrasound. The TI-RADS establish different diagnostic categories with different risks of possible malignancy, comparable with the breast risk assessment tool, Breast Imaging Report (BIRADS) US system. Russ et al [18] included the elastographic aspect of the nodules as one of the 6 diagnostic criteria of a TI-RADS model and obtained high sensitivity and specificity. The study used pooled data from cytological confirmed benign nodules and pathology reports for suspected malignant lesions. The reason behind using TI-RADS is to select the nodules for FNAB in a cost-effective manner, and to emphasize attention on the right nodules referred tobiopsy.

FNAB results are not $100 \%$ accurate, with sensitivity and specificity up to $80 \%$ in very good centers [19] and are considered as a pre-diagnostic method and not as a golden standard diagnostic method [20] due to the sensitivity of $70 \%-85 \%[20,21]$ and also an application rate of $66 \%$ [21]. The FNAB findings reported only $47 \%-55.3 \%$ $[21,22]$ of proven cancers. The rate of false negative and false positive results of FNAB remains a challenge of this diagnostic method [20].

The aim of the present study is to evaluate the differential diagnosis performance of TI-RADS when quantitative strain elastography is considered a diagnostic parameter in the TI-RADS model. In order to avoid confounding factors due to the limited diagnostic availability and diagnostic value of FNAB, we applied TI-RADS analysis in nodules with proved histopathological diagnosis after surgical resection.

\section{Material and methods}

The study was performed in accordance with the ethical guidelines of the Helsinki Declaration and was approved by the Ethics Committee of our Center. Written informed consent was obtained from all patients prior inclusion.

\section{Patients}

The study group included patients with uninodular or polinodular goiter examined in our Elastography Unit between January 2013- June 2014. In this period 432 patients were identified with uninodular and multinodular solid goiter and 174 cases received surgical treatment. Histopathological analysis was performed in all casesand was considered the golden standard diagnosis. Conventional gray scale, Doppler US, and strain elastography (SE) were performed prior surgery by one operator with more than 10 years experience in conventional US and 5 years in elastography, maximum 2 months before surgery. FNAB was performed in more than half of the cases prior surgery (data not analyzed in this study). The surgical indication was made in the presence of large nodules $(>4 \mathrm{ml})$, positive FNAB result (Bethesda IV, V, and VI), compression effects, functional autonomy (defined by suppressed TSH levels), and multinodularity. In cases with multinodular goiter, the larger nodule or the nodule with US malignancy findings was considered in the final statistical analysis.

\section{Ultrasound evaluation}

US and SE were performed using Hitachi Preirus (Hitachi Medical Corporation, Tokyo, Japan) machine with 6-13 MHz linear probe. SE was performed using recommendations of Rago et al [23] with mild external pressure, always checked on the pressure scale, using only 3-4 grade images and loops. The Tsukuba (UenoItoh) classification was used for qualitative analysis of SE [24]. The nodules were classified according to simplified TI-RADS system described by Russ et al [18] (Table I). For each nodule, apart from the qualitative SE score (standard blue red green color map), the strain ration was calculated by comparing the nodule with the surrounding healthy, non nodular, thyroid tissue. Regardless of the color map result, (low stiffness $=$ color map 2; intermediate stiffness = color map 3, and high stiffness $=$ colormap 4 and 5 ) if the strain ratio had a value $\geq 4$, high strain on TI-RADS checklist was considered.

\section{Surgical intervention}

Unilateral lobectomy or total thyroidectomy was performed by the surgeons of our team (FV, SP). In selected cases lymph node excision was also performed. 


\section{Pathology examination}

The histological diagnosis was made in the Pathology Department by the thyroid pathology specialist (MD, $\mathrm{MC})$. Imunohistochemical evaluation, HBME, CH-19, K067 and TTF reactions were performed in selected cases.

\section{Statistical analysis}

Data were collected and analyzed using SPSS v.17 statistical software package (SPSS Inc, Chicago, IL, USA). Clinical and laboratory characteristics of the patients were expressed as mean, standard deviation (SD), median, and range. Prior analysis, variables were tested for normality using Shapiro-Wilk test respectively for homogeneity of variances with Levene's test. Sensitivity, specificity, positive and negative predictive values, and likelihood ration were calculated using TI-RADS 2,
3 for benign lesions, 4A for both benign and malignant lesions, respectively for score $4 \mathrm{~B}$ and 5 for malignant lesions. The diagnostic performance of TI-RADS was evaluated also using the receiver-operating (ROC) curve. The ROC curve represents sensitivity versus 1 -specificity for all possible TI-RADS values for the prediction of thyroid cancer.

\section{Results}

From the total 174 analyzed cases, 29 (16.7\%) were histological proven to be malignant: papillary carcinoma 23 cases, follicular carcinoma 3 cases, and medullar carcinoma 2 cases ( 1 isolated and 1 familial case).

The classification of the thyroid nodules, according to the TI-RADS system, is presented in Table II. The differ-

Table I. Used TI-RADS classification (adapted from Russ et al [18])

\begin{tabular}{|c|c|c|}
\hline TIRADS & Interpretation & Ultrasonographic findings \\
\hline 1 & Normal thyroid findings & - Normal thyroid tissue without any nodular aspect \\
\hline 2 & Constantly benign aspect & $\begin{array}{l}\text { - simple cyst, spongiform nodules } \\
\text { - "white knight" } \\
\text { - isolated macrocalficication, nodular hyperplasia }\end{array}$ \\
\hline 3 & Very probably benign & - no signs of high suspicion, isoechoic or hyperechoic, partial in capsulated \\
\hline $4 \mathrm{~A}$ & Undetermined & - no signs of high suspicion, mildly hypoechoic, encapsulated nodule \\
\hline $4 \mathrm{~B}$ & Suspicious & 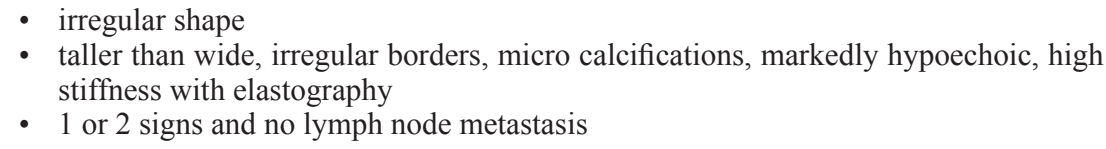 \\
\hline 5 & Highly suspicious & $\begin{array}{l}\text { - } \text { irregular shape/ taller than wide, irregular borders } \\
\text { - micro calcifications } \\
\text { - markedly hypoechoic } \\
\text { - high stiffness with elastography: strain ratio }>4 \\
\text { - } 3 \text { to } 5 \text { signs and/or lymph node metastasis }\end{array}$ \\
\hline
\end{tabular}

Table II. TI-RADS categories and risk of malignancy of the studied thyroid nodules.

\begin{tabular}{lllll}
\hline TI-RADS category & Benign & Malignant & Total & Risk of malignancy (\%) \\
\hline 2 & 15 & 0 & 15 & 0 \\
3 & 84 & 1 & 85 & 1.125 \\
4A & 43 & 3 & 46 & 6.52 \\
$4 \mathrm{~B}$ & 2 & 9 & 11 & 81.81 \\
5 & 1 & 16 & 17 & 94.11 \\
Total & 145 & 29 & 174 & - \\
\hline
\end{tabular}

Table III. TI-RADS categories and diagnostic performance of strain elastography

\begin{tabular}{lllllll}
\hline Method & Sensitivity (\%) & Specificity (\%) & PPV (\%) & NPV (\%) & LR+ & Accuracy \\
\hline TI-RADS 2 & 10.34 & 100 & 100 & 18.23 & - & 25.28 \\
TI-RADS 3 & 57.93 & 96.55 & 98.82 & 31.46 & 10.36 & 58.62 \\
TI-RADS 4A & 29.65 & 89.65 & 93.47 & 20.31 & 2.63 & 39.65 \\
TI-RADS 4B qualitative SE & 31.03 & 98.62 & 81.81 & 97.94 & 22.14 & 87.35 \\
TIRADS 4B quantitative SE & 24.13 & 96.55 & 53.84 & 86.41 & 7.088 & 84.48 \\
TIRADS 5 qualitative SE & 55.17 & 99.31 & 94.11 & 91.71 & 78.71 & 91.95 \\
TIRADS 5 quantitative SE & 44.82 & 97.93 & 8.25 & 89.87 & 21.64 & 89.08 \\
\hline
\end{tabular}

SE - strain elastography, PPV - positive predictive value, NPV - negative predictive value, LR - like hood ration 
Table IV. TI-RADS categories and overall diagnostic performance

\begin{tabular}{llll}
\hline TI-RADS category & Benign & Malignant & Total \\
\hline TI-RADS 2,3,4A & 142 & 4 & 146 \\
TI-RADS 4B,5 & 3 & 25 & 28 \\
Total & 145 & 29 & 174 \\
\hline
\end{tabular}

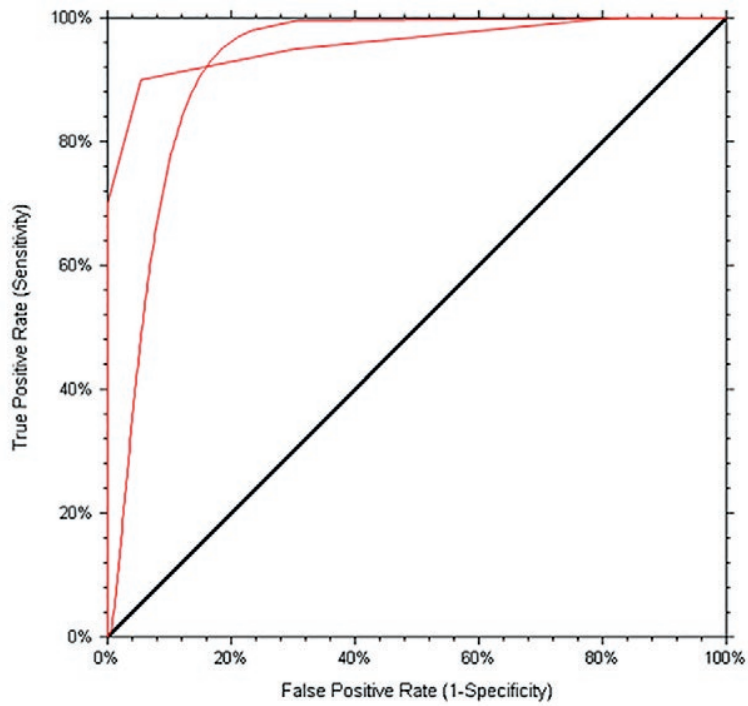

Fig 1. ROC results for the TI-RADS ultrasonography + strain ratio in the diagnosis of thyroid cancer (prevalence of $16.66 \%$ )

ent TI-RADS categories were compared with the pathology report and the malignancy risk was calculated.

Both quantitative and qualitative SE was considered. The sensitivity, specificity, positive predictive value (PPV) and negative predictive value (NPV) and accuracy were calculated. The results are presented in Table III.

Combining TI-RADS 2,3 and 4A as probably benign and TI-RADS $4 \mathrm{~B}$ and 5 as probably malignant, the sensitivity, specificity, positive predictive value, negative predictive value were respectively $97.93 \%, 86.20 \%, 97.26 \%$ and $89.28 \%$. The overall accuracy of the method was $95.97 \%$.

Using quantitative SE results (strain ration as the $6^{\text {th }}$ TI-RADS criteria), the sensitivity and specificity of the TI-RADS model was improved compared with the qualitative strain elastography. The diagnostic value of the TIRADS system in identifying thyroid cancer, when using the quantitative strain ratio as an indicator for stiffness of a lesion, evaluated by the ROC method, is excellent: $\mathrm{AUC}=0.95761$ [0.8424-0.989] 95\%CI (fig 1).

\section{Discussions}

We evaluated the TI-RADS diagnostic performance in differentiating benign from malignant thyroid nodules using a quantitative strain elastography report, as one of the six diagnostic characteristics, comparing with golden standard, pathology report. There are some published papers evaluating the TI-RADS system in the diagnosis of thyroid masses $[16-19,25,26]$ but the pathological confirmation of the nodules was not performed in all the cases. This problem was underlined in a recent published review [27].

The AUC for TI-RADS system was higher in our study (0.9576) comparing to that described in the recent analysis of the conventional TI-RADS model (0.9177) [27]. A similar good diagnostic quality was described by Rust et al [28]. The Russ et al [18] report was the first that used elastography as a criteria in the TI-RADS system. The authors [18] demonstrated that the association of qualitative strain SE, as the $6^{\text {th }}$ criteria in the TI-RADS, increases the sensitivity $(96.7 \%$ versus $92.5 \%)$, but decreases the specificity (11.4\% versus $20 \%$ ) and accuracy (46.7\% versus $56.8 \%$ ), of the diagnostic. Qualitative SE stiffness criteria proved to have a lower accuracy as compared with our result, when quantitative SE stiffness was used. Analyzing the results of elastography alone, the authors obtained a sensitivity of $41.9 \%$, which was lower than in the TI-RADS US system, but the specificity was higher, of $86.4 \%$. These results differ from the results of a recent meta-analysis [13], where the pooled sensitivity and specificity of the SE were $90 \%$, respectively $81 \%$. The low values of sensitivity, in Russ's study, suggest that the elastography evaluation was not clear. This is the reason for using quantitative ES as a TI-RADS criteria instead of the subjective, qualitative ES information.

The sensitivity and specificity our TI-RADS model of the diagnosis is very high compared with other TI-RADS studies $[16,17,28]$. Worthy of mentioning is that, in all these studies, the qualitative, color map scheme was used as a criteria for stiffness. Some authors used the 5 score color map scheme [18], others the 4 scale color map scheme [28]. The definition of increased stiffness was also different, using color map 3-4 [13] or only 4 [18].

The major difference of our study comparing with published studies regarding this problem is the use of quantitative elastography in the TIRADS system. Regardless of the SE color map scheme category 3,4 , or 5 , value dependent on the operator experience, the strain ration is calculated by computer and could decrease the number of false results. In cases where the strain ratio was higher than 4 , we considered the lesion to be stiff and we added a step in the TIRADS category. Using the computer assisted assessment of stiffness of the lesions, we observed excellent diagnostic sensitivity, $86.20 \%$ with high specificity, $97.24 \%$, and the best accuracy of $95.40 \%$. The use of the strain ratio as criteria in the TIRADS system increases 
the confidence of the diagnosis. Clear confirmation of increased strain, is highly suspicious for malignancy. The number of false positive and false negative results was extremely low with the use of the quantitative elastography report instead of the qualitative color map scheme. We had only one false positive result in the TI-RADS category 5 , a case with extended tuberculosis with intrathyroidal nodular lesions. The probability of TI-RADS 2 and 3 nodules to be malignant is extremely low. We had only 1 case classified as TI-RADS 3 with histological structure of micropapillary carcinoma.

The risk stratification of the nodules, according to the TI-RADS value, is similar to the previous published results [25-28]: TI-RADS 2 extremely low cancer risk, less than $1 \%(0 \%$ in our case), very low for TI-RADS 3 cases, less than $5 \%(1.15 \%)$, low for TIRADS 4A cases, less than $10 \%(6.51 \%)$, with extremely high risk for TI-RADS 4B and 5 cases, over $80 \%$ ( $81.81 \%$ respectively $94.11 \%)$. TI-RADS evaluation is described to decrease the number of unnecessary FNAB procedures [16,18,27,28]. When quantitative SE was used, the diagnostic quality did increase without any negative impact on risk stratification quality.

Considering the AACE Guidelines [10] the number of required FNAB in our study would be 169 cases [10]. If the TI-RADS classification is applied, FNAB is necessary in 74 nodules (all 4A, 4B and 5 TIRADS nodules). The decrease is higher compared to the result reported by Horvath et al [16] $43.7 \%$ versus $35 \%$, but comparable with the $42 \%$ decrease in the Moifo et al and Rust et al studies [26,28].

Study limits: not all cases had the FNAB evaluation. Ideally all cases should have been analyzed by the TI-RADS model, FNAB, and pathology report. An increased number of cases is preferable. Further studies are required in order to validate the TI-RADS analysis for daily clinical endocrine practice. Another limit is the lack of the intra-observer and inter-observer agreement. Also, in our analysis we did not take in consideration the clinical aspect, TSH and free-T4 assessment, and results of scintigraphy, aspects with great importance in clinical practice.

\section{Conclusions}

The TI-RADS system, proposed by Russ, with 5 conventional ultrasound parameters and 1 elastography parameter, is a useful tool in the risk stratification of thyroid nodular masses. It may be used forreducing the number of unnecessary FNAB evaluations. Quantitative elastography brings constant, valuable but also clear criteria in the TI-RADS model of risk stratification. Quantitative
SE, as the $6^{\text {th }}$ parameter of TI-RADS system adds diagnostic power to the risk stratification model.

\section{Conflict of interest: none}

\section{References}

1. Vanderpump MPJ. The epidemiology of thyroid diseases. In: Braverman LE, Utiger RE (eds.) Werner and Ingbar's THE THYRIOD: A Fundamental and Clinical text, 9th edn. JB Lippincott-Raven: Philadelphia, 2005: 398-496.

2. Hegedus L. Clinical practice. The thyroid nodule. New Engl J Med 2004; 351: 1764-1771.

3. Vanderpump MP. The epidemiology of thyroid disease. $\mathrm{Br}$ Med Bull 2011; 99: 39-51.

4. Pellegriti G, Frasca F, Regalbuto C, Squatrito S, Vigneri $\mathrm{R}$. Worldwide increasing incidence of thyroid cancer: update on epidemiology and risk factors. J Cancer Epidemiol 2013; 2013: 965212.

5. Papini E, Guglielmi R, Bianchini A, et al. Risk of malignancy in nonpalpable thyroid nodules: predictive value of ultrasound and color-Doppler features. J Clin Endocrinol Metab 2002; 87: 1941-1946.

6. Mathews JD, Forsythe AV, Brady Z, et al. Cancer risk in 680,000 people exposed to computed tomography scans in childhood or adolescence: data linkage study of 11 million Australians. BMJ 2013; 346: f2360.

7. Wulaningsih W, Michaelsson K, Garmo H, et al. Inorganic phosphate and the risk of cancer in the Swedish AMORIS study. BMC Cancer 2013; 24: 257.

8. Tumbridge WM, Evered DC, Hall R, et al. The spectrum of thyroid disease in a community: the Whickham survey. Clin Endocrinol 1977; 7: 481-493.

9. American Thyroid Association (ATA) Guidelines Taskforce on Thyroid Nodules and Differentiated Thyroid Cancer, Cooper DS, Doherty GM, Haugen MD, et al. Revised American Thyroid Association management guidelines for patients with thyroid nodules and differentiated thyroid cancer. Thyroid 2009; 19: 1167-1214.

10. Gharib H, Papini E, Paschke R et al. American Association of Clinical Endocrinologist, Associazione Medici Endocrinologi, and European Thyroid Association Medical Guidelines for Clinical Practice for the Diagnosis and Management of Thyroid Nodules. Endocr Pract 2010; 16 (suppl 1): 1-43.

11. Moon WJ, Baek JH, Jung SL, et al; Korean Society of Thyroid Radiology (KSThR); Korean Society of Radiology. Ultrasonography and the ultrasound-based management of thyroid nodules: consensus statement and recommendations. Korean J Radiol 2011; 12: 1-14.

12. Levine RA. Current guidelines for the management of thyroid nodules. Endocr Pract 2012; 18: 596-599.

13. Sun J, Cai J, Wang X. Real time elastography for differentiation of benign and malignant thyroid nodules: a metaanalysis. J Ultrasound Med 2014; 33: 495-502.

14. Cantisani V, Grazhdani H, Drakonaki E, et al. Strain USelastography for the characterization of thyroid nodules: 
Advantages and Limitation. Int J Endocrinol 2015; 2015: 908575.

15. Dudea SM, Botar-Jid C. Ultrasound elastography in thyroid disease. Med Ultrason 2015; 17: 74-96.

16. Horvath E, Majilis S, Rossi R, et al. An ultrasonogram reporting system for thyroid nodules stratifying cancer risk for clinical management. J Clin Endocrinol Metab 2009; 94: 1748-1751.

17. Kwak JY, Han KH, Yoon JH, et al. Thyroid imaging reporting and data system for US features of nodules: a step in establishing better stratification of cancer risk. Radiology 2011; 260: 892-899.

18. Russ G, Royer B, Bigorgne C, Rouxel A, Bienvenu-Perrard M, Leenhardt L. Prospective evaluation of thyroid imaging reporting and data system on 4550 nodules with and without elastography. Eur J Endocrinol 2013; 168: 649-655.

19. Jo VY, Stelow EB, Dustin SM, Hanley KZ. Malignancy risk for fine-needle aspiration of thyroid lesions according to the Bethesda System for Reporting Thyroid Cytopathology. Am J Clin Pathol 2010; 134: 450-456.

20. Wang CC, Friedman L, Kennedy G, et al. A large multicenter correlation study of thyroid nodule cytopathology and histopathology. Thyroid 2011; 21: 243-251.

21. Giard RW, Hermans J. Use and accuracy of fine-needle aspiration cytology in histologically proven thyroid carcinoma: an audit using a national anthology database. Cancer 2000; 90: 330-334.
22. Mistry SG1, Mani N, Murthy P. Investigating the value of fine needle aspiration cytology in thyroid cancer. J Cytol 2011; 28: 185-190.

23. Rago T, Santini F, Scutari M, Pinchera A, Vitti P. Elastography: new developments in ultrasound for predicting malignancy in thyroid nodules. J Clin Endocrinol Metab 2007; 92: 2917-2922.

24. Itoh A, Ueno E, Tohno E, et al. Breast disease: clinical application of US elastography for diagnosis. Radiology 2006; 239: 341-350.

25. Cheng SP, Lee JJ, Lin JL, Chuang SM, Chien MN, Liu CL. Characterization of thyroid nodules using the proposed thyroid imaging reporting and data system (TI-RADS). Head and Neck 2013; 35: 541-547.

26. Moifo B, Takoeta EO, Tambe J, Blanc F, Fotsin JG. Reliability of Thyroid Imaging Reporting and Data System (TIRADS) Classification in Differentiating Benign from Malignant Thyroid Nodules. OJRad 2013; 3: 103-107.

27. Wei X, Li Y, Zhang S, Gao M. Thyroid imaging reporting and data system (Ti-RADS) in the diagnostic value of thyroid nodules: a systematic review. Tumor Biol 2014; 35 : 6769-6776.

28. Friedrich-Rust M, Meyer G, Dauth N, et al. Interobserver agreement of Thyroid Reporting and Data System (TIRADS) and strain elastography for assessment of thyroid nodules. PLoS One 2013; 8: e77927. 